\title{
Jahrestagung des Arbeitskreises Kinderurologie 20. bis 21. Januar 1995
}

\author{
S. Schumacher \\ Mainz
}

Dr. Humpl aus der Kinderklinik Mainz sprach in seiner State-of-the-Art Lecture über den aktuellen Wissensstand auf dem Gebiet des Neuroblastoms. Die jährliche Inzidenz dieses Tumors liegt in den Industrienationen bei 9-10 Fällen pro eine Million Kinder unter dem 15. Lebensjahr. Bei Kindern unter dem ersten Lebensjahr ist die Inzidenz höher. Das Durchschnittsalter der Erstmanifestation liegt bei 22 Monaten. Die Ätiologie ist unbekannt. Die malignen Zellen entstehen im allgemeinen in primitiven, embryonalen, sympathischen Neuroblasten. Dabei sind Hauptmanifestationsorgane Paraganglien wie das Nebennierenmark oder das Zuckerkandl-Organ und alle Regionen, in denen sympathisches Nervengewebe gefunden werden kann. Das Neuroblastom kann sich intraoder extraspinal ausbreiten. Neben lokaler Invasivität finden sich metastatische Prozesse in Lymphknoten, Knochenmark, Skelett, Leber, Haut und Hoden. Das klinische Bild dieses Tumors wird durch die primäre Lokalisation, Invasivität und Metastasenaktivität geprägt und ist daher nie einheitlich.

$90 \%$ der Neuroblastomzellen zeigen erhöhte oder abnorme Produktion, Sekretion und Katabolismus von Katecholaminen. Daher ist die Ausscheidung von Homovanillinsäure, Vanillinmandelsäure und Dopmain beim Neuroblastom sowohl differentialdiagnostisch als auch prognostisch von großer Bedeutung. Differentialdiagnostisch spielen Wilms-Tumor, Lymphom, Hepatosplenomegalie und Rhabdomyosarkom eine bedeutende Rolle. Als gesichert ist die Diagnose anzusehen, wenn eine eindeutige pathologische Diagnose von entnommenem Gewebe lichtmikroskopisch gestellt werden kann oder Knochenmarksaspiration oder Schädelbiopsie eindeutig Tumorzellen enthalten und erhöhte Serum- oder Urinkatecholamine oder deren Metaboliten nachgewiesen werden können. Die Bestimmung der neuronspezifischen Enolase (NSE) ist ein guter Verlaufsparameter der Erkrankung und deren Therapie. Unspezifisch ist hier eine Verlaufskontrolle der LDH oder des Ferritins, das bei ausgedehnten Befunden signifikant erhöht ist.

Neben CT und MRT hat der MIBG-Scan zur Lokalisation von Primärtumor und Metastasen sowie zur Verlaufskontrolle klinische Bedeutung. Des weiteren hat die $99 \mathrm{~m}$ Tc-Szintigraphie zur zusätzlichen Differenzierung von Skelettprozessen Bedeutung. Therapeutisch sind die Kombination aus Chemotherapie, operativem Procedere und Radiotherapie jeweils abzuwägen. Prognostischen Charakter hat das primäre Tumorstadium, das Alter des

Akt. Urol. 26 (1995) 288-293

(C) Georg Thieme Verlag Stuttgart · New York
Patienten und die Tumorlokalisation. 80-90\% der Patienten im Tumorstadium Evans I, II und IV-S sowie $50-60 \%$ der Patienten im Tumorstadium III können geheilt werden. Die Prognose im Stadium IV ist hingegen schlecht.

Abramovic et al. unterstrichen die Bedeutung der Altersstufe des Kindes als wichtigsten prognostischen Parameter. Die Therapieerfolge im ersten Lebensjahr waren deutlich besser. Aufgrund meist ausgedehnter Tumorbefunde bei Diagnosestellung im eigenen Patientengut werden als Screening die Bestimmung von Vanillinmandelsäure und Homovanillinsäure im Urin im sechsten Lebensmonat empfohlen.

Zöller et al. stellten eine modifizierte postoperative Polychemotherapie mit Carboplatin, Etoposid, Ifosfamid und Epirubicin beim fortgeschrittenen oder metastasierten Wilms-Tumor aufgrund schlechter Therapieerfolge des bisherigen SIOP-9-Protokolls vor. Diese Therapie zeigte nach ersten Ergebnissen an 3 Patienten keine Verbesserungen und wurde im Hinblick auf die erhebliche Knochenmarks-Toxizität als sehr kritisch angesehen. Der fehlende Effekt von Ifosfamid auf Wilms-Tumoren wurde vom Auditorium diskutiert.

Bei der Fragestellung nach diagnostischen Parametern der Wilms-Tumoren wurde die Bedeutung des Insulin-like-growth factor-II (IGF-II) von der Arbeitsgruppe Nelde untersucht. Sie kamen zu dem Ergebnis, daß das IGF-II-Protein sowohl in tumortragendem als auch in tumorfreiem Nierengewebe zu finden war, was die mögliche tumorgenetische Rolle unklar läßt. IGF-II kann ihrer Ansicht nach nicht als diagnostischer Marker gewertet werden.

$K$. Hohenfellner et al. untersuchten retrospektiv das biologische Verhalten von Wilms-Tumoren an 39 Patienten mit Hilfe von Durchfluß- und Bildzytometrie sowie der AG-NOR(Argyrophylic staining of nuclear organizer region-associated proteins)-Diagnostik. Als Methode der Wahl wurde die Flowzytometrie gewertet, da diese den anderen beiden Verfahren überlegen war. Bei histologisch schwierig klassifizierbaren triphasischen Nephroblastomen kann sie zusätzlich hilfreich sein. Wichtigste prognostische Parameter sind weiterhin das Tumorstadium und die Histologie.

An den Fällen von 6 Kindern wurden von Vögeli et al. Diagnose- und Therapiemöglichkeiten sowie der Verlauf einer BK-Polyomavirus induzierten hämorrhagischen Zystitis nach Knochenmarkstransplantation aufgezeigt. Differentialdiagnostisch waren zusätzlich eine 
Thrombozytopenie, eine bakterielle sowie eine chemisch induzierte Zystitis zu berücksichtigen. Nach erfolgtem Virusnachweis im Urin und/oder Biopsien der umschriebenen polypös veränderten Blasenschleimhaut beschrieben die Autoren bei persistierender Blutung die Notwendigkeit eines therapeutischen Procederes mit Zystoskopie, Einlage einer suprapubischen Zystostomie und endoskopischer Laservaporisation, wobei die mechanische Manipulation vom Auditorium sehr kritisch diskutiert und die Frage nach möglichen uroprotektiven Substanzen aufgeworfen wurde. 2 Kinder im Alter von 5 und 9 Jahren verstarben an einer Graft versus host reaction.

Unter diesem ersten großen Programmpunkt wurden auch einige uro-onkologische Fallvorstellungen präsentiert.

Die Arbeitsgruppe Weingärtner schilderte den Fall eines 5jährigen Mädchens mit der Kombination aus Wilms-Tumor, Fallotscher Tetralogie und Dysmelie, wobei die Frage nach einem neuen genetisch fixierten Syndrom aufgeworfen wurde. Der jüngere Bruder leidet ebenfalls an einer Fallotschen Tetralogie. Beide Eltern sind gesund. Ein engmaschiges Screening des Bruder zur Früherkennung eines Wilms-Tumors wird durchgeführt. In der Diskussion wurde allerdings vom Auditorium kritisch angemerkt, daß es sich bei dem Holt-Oram-Syndrom (Kombination aus Fallotscher Tetralogie und Dysmelie) im Gegensatz zum in ca. $30 \%$ der Fälle scheinbar autosomal dominant vererbten Gendefekt beim Wilms-Tumor um eine Hemmungsmißbildung handelt.

Hermanns et al. schilderten den Fall eines 8jährigen Jungen mit großem retroperitonealen Tumor, der sich histologisch als juvenile desmoide Fibromatose darstellte und nach zuvor im Ausland durchgeführter Radiatio radikal operiert wurde. Das Wachstum ist lokal invasiv. Die Ätiologie ist unklar.

Latal et al. stellten urologische Komplikationen nach radikaler Tumorentfernung eines zuvor mit Radiatio und Chemotherapie behandelten embryonalen Rhabdomyosarkoms der linken Abdominalwand vor, die nach zahlreichen rekonstruktiven Eingriffen im Bereich der harnableitenden Wege in einer Psoas Hitch-BoariKombination rechts bei Verlust der linken Niere endeten. Die Obstruktion als Spätfolge von Radiatio und Chemotherapie wurde nach Auffassung der Autoren falsch eingeschätzt.

Des weiteren wurde von Schumacher et al. der Fall eines 7jährigen Mädchens vorgestellt, das nach der Resektion einer Ureterozele ein nephrogenes Adenom der Blase entwickelte. Häufigste prädisponierende Faktoren bei der Entstehung dieses benignen Tumors im Kindesalter wurden in der operativen Manipulation an Blase und/oder Harnleiter sowie chronisch rezidivierenden Harnwegsinfekten gesehen. Therapeutisch wurde bei häufigster Tumorlokalisation in der Blase eine transurethrale Resektion mit späterer Nachresektion bei hoher Rezidivrate empfohlen.

Günther berichtete über ein unreifes Teratom des Hodens beim 8 Wochen alten Säugling. Vom Audi- torium wurde hier die Meinung vertreten, daß ohne Vorliegen von Metastasen keine Chemotherapie erfolgen sollte.

Conrad et al. zeigten den Fall eines 7jährigen Kindes mit paravesikal gelegenem zystischen Lymphangiom. Differentialdiagnostisch wurden ein Blasendivertikel, ein persistierender Urachus, eine Beckenniere sowie eine Echinokokkuszyste diskutiert. wahl freier Themen.

Weiter ging es im Programm mit einer Aus-

Stein et al. berichteten über den Langzeitverlauf nach Anlage eines isoperistaltischen, antirefluxiven Kolon-Conduits bei insgesamt 112 Kindern und Heranwachsenden. Indikationen zu operativen Maßnahmen ergaben sich meist aufgrund neurogener Blasenentleerungsstörungen. Ein aktuelles Follow up wurde bei $87 \mathrm{~Pa}$ tienten erhoben, die mittlere Follow up-Zeit lag bei ca. 15 Jahren. Frühkomplikationen mit mechanischem Ileus und Pyonephrose wurden bei 4,5\% der Patienten beschrieben. Späte Komplikationen betrafen 26 Patienten. Hier waren Pyelonephritiden, Steine, Stoma- und Harnleiterstenosen, Ileus und metabolische Azidosen die Hauptursachen. Lag präoperativ eine ausgeprägte Dilatation des oberen Harntraktes vor, kam es in keinem Fall zur Normalisierung des oberen Harntraktes. War das Serumkreatinin bereits präoperativ erhöht, so normalisierte sich dieses nur bei einem Drittel der Patienten. Kein Patient mit initial normalen Kreatininwerten entwickelte ein chronisches Nierenversagen.

Heute wird die Indikation zum Kolon-Conduit im Kollektiv der Kinder mit neurogenen Blasenentleerungsstörungen durch $\mathrm{CIC}$, anticholinerge Therapie, Blasenaugmentation und kontinente Harnableitungen seltener gestellt. Indikationen beschränken sich auf eine grenzwertige Nierenfunktion, psychosoziale und/oder motorische Störungen sowie Kinder unter 3 Jahren. Besonders bei letzteren Kindern ist das Kolon-Conduit als intermediäre Form der Harnableitung zu sehen und kann später in kontinente Formen konvertiert werden. Eine sich verselbständigende Pyelonephritis mit Steinbildung und weiterer Einschränkungen der Nierenfunktion ist aufgrund der verspäteten Indikationsstellung zu sehen. In der Diskussion wurde hervorgehoben, daß eine Konversion bei unauffälligem oberen Harntrakt in der Regel erfolgreich sei.

Die Arbeitsgruppe Sperling stellte ein katheterisierbares Appendixstoma zur Therapie der neurogenen Stuhlinkontinenz bei 6 MMC-Patienten vor. Keiner der Patienten, die unter anticholinerger Therapie bzw. sauberem Einmalkatheterismus urinkontinent waren, konnten eine soziale Stuhlkontinenz erreichen. Die Appendix wurde in die Taenia libera des Kolons eingebettet und im Nabel oder dem rechten Unterbauch ausgeleitet. Selbsttätige antegrade Darmspülungen mit $500-1500 \mathrm{ml}$ NaCl oder Golitelylösung lassen eine soziale Stuhlkontinenz von mindestens 2 Tagen erreichen. Kritisch wurde aus dem Auditorium angemerkt, daß die zu erwartenden Ergebnisse bei inkontinentem Analsphinkter als gut, bei spastischem Analsphinkter allerdings eher als fraglich einzuschätzen seien. 
Kallerhoff et al. berichteten über Ergebnisse der Durchflußzytophotometrie nach Hodentorsion. Hierbei konnte der mit der Chromosomenzahl korrelierende DNS-Gehalt der Zellkerne gemessen werden. Im Histogramm zeigte sich eine Abnahme der Spermatiden und Spermien um $20 \%$ innerhalb der ersten sechs Stunden, während die Parenchymzellen und diploiden Zellen der Spermiogenese in dieser Zeit zunahmen. Bei zunehmender Torsionszeit kam es zu vermehrtem Auftreten von Zellen mit von der Norm abweichendem DNS-Gehalt. Nach Kühlung zeigte sich eine verbesserte Ischämietoleranz. Diskutiert wurde die klinische Relevanz mit langsamem intraoperativen Aufwärmen des Hodens und der präoperativen Hodenkühlung, falls diese vom Patienten toleriert wird.

Auch dieser freie Themenbereich wurde durch Einzelfallbeispiele ergänzt.

Sauvage stellte Ergebnisse von 2 Patienten mit Blasenekstrophie vor, die nach primärer Korrektur mit Verschluß der Blase, Urethral- und Sphinkterrekonstruktion sowie ggf. Deckung des Hautdefektes mit Skrotalhaut eine primäre Kontinenz im Alter von 2-3 Jahren erreichten. Mehrere Eingriffe im Bereich der äußeren Genitale konnten eine korrekte Gliedlänge und Meatusposition erbringen. Heute im Alter von 19 und 22 Jahren sind beide über 3 Stunden kontinent und haben ein normales Sexualleben. Gezeigt werden sollte, da $\beta$ durch den primären Verschluß eine Kontinenz erreicht und auf eine Beckenosteotomie verzichtet werden kann.

Die Gruppe Schönberger stellte 5 Patienten im Alter zwischen 5 und 57 Jahren mit retrokavalem Ureter vor, von denen vier symptomatisch waren. Diagnostisch wurde die Kombination aus Sonographie, IVP (flaue KMAnfärbung), CT und retrograder Harnleiterdarstellung, ggf. in Kombination mit einer Kavographie aufgezeigt. Therapeutisch wurde in allen Fällen eine trans- oder retroperitoneale Harnleiterdurchtrennung durchgeführt. Diskutiert wurde die frühzeitige Diagnosestellung durch den Einsatz der Sonographie.

Gerharz et al. schilderten den Fall eines 11jährigen Jungen mit computertomographisch bauchwandinfiltrierender eosinophiler Zystitis. Zystoskopie, Biopsie und Serumlabordiagnostik (BB, Immunelektrophorese) sicherten die Diagnose. Differentialdiagnostisch waren Blasentumoren des Kindesalters sowie Zystitiden unterschiedlichster Genese zu diskutieren. Therapeutisch wurde nach Antibiotika- und Glukokortikoid-Medikation eine Restitutio nach 5 Monaten erreicht.

Filipas und Kollegen berichteten über einen 9jährigen Jungen mit tumorbildender Urocystitis cystica, der aufgrund einer Enuresis diurna et nocturna in Behandlung war. Urodynamisch zeigte sich eine passive Urininkontinenz bei kleinkapazitärer Blase. Trotz hypotonem Urethraprofil waren erhöhte Restharnmengen nachzuweisen. Urethrozystoskopisch imponierte ein polypartiger Tumor präsphinktär. Nach Resektion kam es viermal zum Rezidiv innerhalb eines Jahres.

Von der Arbeitsgruppe Heinz wurde ein 20 Monate altes Kleinkind mit Pseudotumor der Harnblase vorgestellt. Der sonographische Zufallsbefund wurde durch eine TUR-Blase therapiert. Histologisch zeigte sich eine unspezifische Entzündung. Bei einem 17 Monate alten Kleinkind mit sonographisch identischem Befund war ein Therapieerfolg nach einer Antibiotikagabe über 4 Wochen zu verzeichnen. Diskutiert wurde über die Differentialdiagnose des Rhabdomyosarkoms der Harnblase.

Humke und Kollegen zeigten an 6 Fallbeispielen, daß die Kenntnis der Bandbreite renaler Hypertonieformen besonders im Kindesalter von großer Bedeutung ist. Hierbei sind die sekundären Hypertonieformen, insbesondere renaler Genese (renoparenchymatös, -vaskulär) häufigste Ursache. Diagnostisch kommt der seitengetrennten Plasmareninaktivität im Nierenvenenblut und der Angiographie eine entscheidende Rolle zu. Das aufgezeigte therapeutische Spektrum umfaßte neben vaskulären Eingriffen (splenorenaler Arterienbypass, superselektive Katheterembolisation bei arterio-venöser Fistel) die Nephrektomie bzw. Segmentresektion bei Ask-UpmarkNiere.

Der erste Teil des zweiten Tages behandelte die Obstruktion des Harntraktes. Prof. Chevalier aus Charlottesville, Virginia, schilderte in seiner hervorragenden State-of-the-Art Lecture die Bedeutung der unilateralen Obstruktion der harnableitenden Wege im Hinblick auf eine gestörte Nierenentwicklung und hieraus resultierender Nierenfunktionseinschränkung. Je frühzeitiger eine unilaterale Obstruktion auftritt, um so gravierender ist die Schädigung der betroffenen Niere mit gleichzeitig vermehrter Kompensationsleistung der Gegenniere. Untersuchungen am Meerschweinchen haben die Bedeutung der frühen fetalen Obstruktion mit konsekutiver renaler Dysplasie und der Abnahme funktionsfähiger Nephrons gezeigt. Das Renin-Angiotensin-System wird vermehrt aktiviert, was mit vaskulärer und glomerulärer Kontraktion verbunden ist. Die Ergebnisse verschiedener Experimente haben die enge Korrelation der renalen Ischämie mit dem renalen Wachstum gezeigt. Beim "Guinea-Pig" resultiert aus der frühzeitigen Beseitigung der Obstruktion zwar eine Erhöhung des Blutstroms der ipsilateralen Niere, dennoch bleibt das renale Wachstum reduziert. Angiotensin II ist jedoch als Wachstumsfaktor nicht direkt für die Wachstumsverzögerung verantwortlich. Die chronische Obstruktion verursacht eine interstitielle Fibrose, die ihrerseits zu einem verminderten Nierenwachstum beiträgt. Die neonatale Obstruktion verzögert die Freisetzung von epidermalen Wachstumsfaktoren und prolongiert die Ausschüttung von peritubulärem "Alpha smooth muscle actin". Auch dieses weist auf eine verzögerte renale Entwicklung bei unilateraler Obstruktion hin. Der Wachstumsfaktor "Transforming Growth Factor-Beta-1" stimuliert die Synthese extrazellulärer Matrix und hemmt gleichzeitig deren Abbau. Damit spielt dieser Faktor eine Rolle bei der Entwicklung der interstitiellen Fibrose. Bei einseitiger Obstruktion kommt es zu einem signifikanten Anstieg. Angiotensin II kann diesen Faktor stimulieren. Der entwicklungsbedingte Anstieg des "Epidermal Growth Factor" unterliegt ebenfalls den Einflüssen einer Obstruktion und ist bei neugeborenen Ratten mit Obstruktion deutlich vermindert. Ungeachtet der Obstruktion wird die Ausschüttung durch Angiotensin AT 1-Rezeptoren reguliert. Die renale „Apoptosis“ (ein generalisiert determinierter Zelluntergang) ist bei der unilate- 
ralen Obstruktion der neugeborenen Ratte signifikant erhöht, was zu einem verminderten DNA-Gehalt und einer Wachstumsverzögerung der neonatalen Niere beitragen kann. „Clusterin“, ein Glykoprotein mit protektiven Eigenschaften besonders tubulärer Zellen, ist unter anderem obstruktionsbedingt erhöht. Das renale Wachstum sowie die Entwicklung werden durch komplexe Interaktionen aufgezeigter Faktoren mit Zellproliferation, Zelldestruktion und extrazellulärer Matrix reguliert.

Fichtner et al. stellten Ergebnisse des Einflusses von Oxybutinin auf das Nierenbeckendruckprofil im Tiermodell mit angeborener unilateraler Hydronephrose und erhöhten pelvinen Druckwerten vor. Nicht medikamentös behandelte Ratten zeigten dabei erhöhte Nierenbeckendrucke, die bei hoher Diureserate den korrespondierenden Blasendruck überstiegen. Tiere, die mit Oxybutinin behandelt wurden, zeigten hingegen deutlich niedrigere pelvine Druckprofile, die bei allen Diureseraten kleiner als der korrespondierende Blasendruck waren. Diskutiert wurde die klinische Relevanz unter Hinweis auf die fehlenden cholinergen Rezeptoren im oberen Harntrakt des Menschen. Der spasmolytische Wirkungsmechanismus des Oxybutinins wurde hervorgehoben.

Beetz et al. berichteten über den Stellenwert der antibiotischen Infektprophylaxe bei Obstruktion der oberen Harnwege im Säuglingsalter. 1\% aller Neugeborenen lassen im Screening pathologische Befunde erkennen, wobei es sich meist um primäre Megaureteren sowie ureteropelvine Stenosen handelt. $80 \%$ dieser Patienten wurden im ersten Lebensjahr diagnostiziert. Ziel der medizinischen Betreuung sind die Vermeidung von Parenchymschäden und deren Folgeerkrankungen. Aus einem Kollektiv von 203 Kindern mit Subpelvinstenose entwickelten $14 \%$ eine Pyelonephritis, davon $69 \%$ vor Diagnosestellung. 3 Kinder entwickelten unter antibiotischer Dauerprophylaxe einen Infekt. Von 50 Kindern mit Megaureteren hatten $22 \%$ eine Pyelonephritis, davon 81 \% vor Diagnosestellung. Ein Patient entwickelte unter antibiotischer Prophylaxe einen Infekt. In der Literatur wird bei Dilatation des oberen Harntraktes meist eine antibiotische Prophylaxe empfohlen. Die Infektprophylaxe ist gerade im frühen Säuglingsalter bei ureteropelvinen Stenosen und Megaureteren sinnvoll, da die Urosepsisgefahr in dieser Altersgruppe häufiger ist als im späten Kindesalter. Im eigenen Kollektiv wird bei primär obstruktivem Megaureter in den ersten 6 Monaten sowie bei Subpelvinstenose mit einer sonographisch gemessenen Nierenbeckenweite von mehr als $10 \mathrm{~mm}$ oder szintigraphisch nachgewiesener Abflußbehinderung eine Infektprophylaxe durchgeführt. Diskutiert wurden die unterschiedlichen Meinungen zum Stellenwert der Infektprophylaxe.

Die Arbeitsgruppe Weitzel berichtete über die Inzidenz urologischer Operationen nach postnatalem Nierenscreening bei 15794 Neugeborenen. Die Inzidenz der Operationen lag bei $0,17 \%$ und hat durch das Screening nicht zugenommen. $87,5 \%$ der operierten Kinder wurden als Risikopatienten erkannt. Die Häufigkeit der Nierenbeckenplastiken lag bei $0,06 \%$, die der Harnleiterneueinpflanzungen bei $0,02 \%$. Das Screening hatte einen hohen Vorhersagewert für operationsbedürftige Erkrankungen im Säuglings- und Kleinkindesalter. Es dient der
Verminderung symptomatischer Harnwegsinfekte, der Veränderung der OP-Indikation sowie der Verbesserung der Prognose.

Das Themengebiet der Reflux-Krankheit wurde ebenfalls in drei Vorträgen behandelt.

Krause et al. stellten die operativen Ergebnisse von 228 Patienten vor, die einen vesikorenalen Reflux hatten und in der Technik von Lich-Gregoir versorgt wurden. 74 Kinder wurden einzeitig bilateral operiert. Bei 16 Kindern kam es zu perioperativen Komplikationen, die in 2 Fällen mit Urinom und einmal mit Ureterobstruktion eine invasive Behandlung erforderlich machten. Unterschiede zwischen unilateraler und einzeitig bilateraler Operation fanden sich nicht. Bei 3 von 165 nachuntersuchten Patienten fielen nach 3 bis 5 Monaten postoperativ Blasenentleerungsstörungen auf. Als Ursache wurde bei dem einzigen einzeitig bilateral operierten Kind dieser Gruppe ein Blasendivertikel gefunden. Einmal war eine Rezidivoperation, einmal eine Ureterneuimplantation bei Stenose erforderlich. Hinweise auf eine erhöhte Komplikationsrate bei einzeitig bilateraler Operation ergaben sich nicht. Dieses Statement wurde allerdings sehr kritisch aufgrund bekannter Komplikationen, wie die Entwicklung einer neurogenen Blasenentleerungsstörung, diskutiert und die einzeitig bilaterale Operation von verschiedenen Zentren abgelehnt.

Die Prävalenz der arteriellen Hypertonie bei Kindern mit erfolgreich operiertem primären vesikorenalen Reflux war Gegenstand einer retrospektiven, an 1060 Kindern durchgeführten Untersuchung. Steffens et al. berichteten über einen Operationserfolg von $96 \%$, das mittlere Follow up lag bei 17 Jahren. 10,5\% der Patienten mit Nierennarben entwickelten im Alter von 12 bis 14 Jahren eine renale Hypertonie. Die Parenchymnarben sind Voraussetzung der Hypertonieentstehung. Es empfehlen sich bei diesen Patienten jährliche Blutdruckkontrollen, nephrourologische Untersuchungen und bei eingeschränkter Nierenfunktion oder Refluxnephropathie sowie Hyperreninämie im Nierenvenenblut ggf. die Nephrektomie.

Sigel stellte die genetische Systematik und Morphologie der vesikorenalen Refluxkrankeit vor. Es erfolgt eine Klassifikationseinteilung in die Gruppen A und B, wobei A weiter in 5 Untergruppen eingeteilt wurde und B die postnatal additiven Veränderungen durch Infekte berücksichtigt. Beim meist einfachen Reflux liegt eine autochthon trigonale desorganisierte Uromorphologie vor, aus der eine renale Hypodysplasie resultiert. Bei den selteneren Formen des komplizierten Reflux führt beispielsweise die Obstruktion im ersten Trimenon zur urorenalen Hypodysplasie. Ein Beispiel ist das Valve-Syndrom. Die Refluxkrankheit beim Valve-Syndrom geht in $40 \%$ mit einer rein obstruktiven Nephropathie und in $20 \%$ mit einer Kombination aus refluxiver und obstruktiver Nephropathie einher.

Die Sitzung über das äußere Genitale wurde durch die sehr gute State-of-the-Art Lecture von Prof. Woodhouse, London, eingeleitet. Er schilderte die sexuellen und reproduktiven Konsequenzen bei angeborenen 
Anomalien des Urogenitaltraktes. Bei der männlichen Blasenekstrophie wurden besonders auf die Veränderungen des äußeren Genitale mit Penisdeviation und veränderter Anatomie der Corpora hingewiesen. Korrigierende Verfahren nach Nesbit oder Cantwell-Ransley wurden vorgestellt. Zur suprapubischen Narbenkorrektur eignen sich Verschiebelappenplastiken der haartragenden Haut. Seine Untersuchungen haben die stark ausgeprägte Sexualität des männlichen Geschlechtes belegt. Hier besteht zumeist eine feste Partnerschaft. Bei den Frauen hingegen gibt es andere Probleme wie zum Beispiel Lageveränderungen im Bereich des äußeren Genitale. Vorgestellt wurde hier eine Technik der Uterusfixation mit einer Goretexschlinge am Os sacrum bei manifestem Uterusprolaps, die allerdings erst nach Abschluß der Familienplanung durchgeführt werden sollte.

Als mögliche Ursachen eines "Mikropenis“ wurden z. B. der hypogonadotrope Hypogonadismus $(40 \%)$, die gemischte Gonadendysgenesie oder der männliche Pseudohermaphroditismus aufgezeigt. Die psychologische Entwicklung dieser Patienten wurde an 12 Fällen kontrolliert. Dabei war die Hälfte der Patienten völlig normal entwickelt. Alle zeigten ein heterosexuelles, männliches Verhalten bei normaler Geschlechtsidentifikation. Bei $76 \%$ war die sexuelle Aktivität unauffällig. Unter Berücksichtigung sexueller Komponenten ist ein normales Leben möglich. Diskutiert wurde über die Ejakulationsfähigkeit bei Epispadie- und Blasenekstrophiepatienten. Hier wurde die bekannte Tatsache hervorgehoben, daß es oftmals über Stunden zum permanenten Tröpfeln des Ejakulats aus einer rekonstruierten Harnröhre oder nicht rekonstruierten Öffnungen kommt. Häufig wird aber auch ein permanentes Tröpfeln durch den Abfluß von Prostatasekret ohne begleitende Ejakulation gesehen.

Krege et al. schilderten den Fall eines $11 / 2$ jährigen Jungen, der nach ritueller Zirkumzision eine Verletzung einer Megalourethra erlitt. Bei $2 / 3$ der Fälle mit Megalourethra besteht eine Hypoplasie des Corpus spongiosum, die seltenere fusiforme Variante beinhaltet zusätzlich eine Hypoplasie der Corpora cavernosa. Die Ätiologie ist unklar. Die penile und glanduläre Harnröhre sind angelegt, wobei die Kanalisation in der 6. Embryonalwoche gestört ist. Bei diesem Patienten lag eine Sonderform vor, da die bereits zuvor rekonstruierte Harnröhre nach proximal über ca. $1,5 \mathrm{~cm}$ hypoplastisch war („Megameatus mit intakter Vorhaut"). Es folgte eine Harnröhrenrekonstruktion mit urethralem Schwenklappen sowie bei Penisdeviation eine Korrektur nach Nesbit.

Günther berichtete über das Schicksal eines Jungen, der mit seit dem Vortag bestehenden beidseitigen Hodenschmerzen, bläulicher Skrotalhaut mit Hautemphysem und inguinal palpablen Lymphknoten zur stationären Aufnahme kam. Die Entzündungsparameter im Serum waren hoch. Eine beidseitige Hodenfreilegung zeigte eine gelblich-weiße Verfärbung beider Hodenhüllen. Es ergab sich bei der histologischen Aufarbeitung eine subtunikale Gewebeeiterung mit Arealen von Nekrosebildung. Mit dem Ziel der Kindesmißhandlung wurde dem Kind vom Vater am Vortag eine intraskrotale Injektion mit dem Computerreinigungsmittel TMTP-35 (tetrachlorkohlenstoffhaltig) verabreicht.
Weit gefächert war erneut die operative Strategie bei der Hypospadie, letzter Themenschwerpunkt des Treffens.

Sauvage stellte dabei ein einzeitiges Verfahren mit kompletter Wiederherstellung der Eichel und der Vorhaut bei distalen Formen vor. Dabei wurde einerseits eine Eichelplastik, andererseits eine Harnröhrenkorrektur mit distal gestieltem dreieckigen Hautlappen von der Ventralseite des Penis durchgeführt. Bei 119 operierten Kindern, meist jünger als 2 Jahre, gab es keine perioder frühen postoperativen Komplikationen. Die Vorhaut war in 109 Fällen geschlossen, der Neomeatus in $74 \%$ orthotop. 10 Fisteln und 2 Phimosen wurden beschrieben.

Haberlik berichtete über Ergebnisse der Operation nach Beck an 41 Patienten, die mit Mobilisierung der vorderen Urethra verbunden ist. Interessant war hier die Unterspritzung mit Epinephrin. Das durchschnittliche Op-Alter lag bei 3 Jahren. Es wurde über gute funktionelle und kosmetische Ergebnisse berichtet. Die Komplikationsrate wurde mit $10 \%$ angegeben und umfaßte im wesentlichen 8 Meatusstenosen, von denen 3 in Narkose und der Rest zu Hause bougiert wurden, sowie 5 Narbenbildungen. Die Langzeitbougierung wurde sehr kritisch diskutiert.

Die Frage nach einer Notwendigkeit der Meatusverlagerung an die Glansspitze bei anteriorer Hypospadie wurde von Fichtner und Kollegen aufgeworfen. Sie führten bei 500 „normalen“ Erwachsenen eine exakte Bestimmung der Meatusposition durch. Bei 55\% der Patienten war der Meatus im vorderen Glansdrittel lokalisiert. In $13 \%$ der Fälle fanden sich inzidentelle Hypospadien mit Meatuslokalisation im proximalen Glansdrittel. Nur $40 \%$ dieser Patienten waren sich irgendeiner Abnormalität bewußt. Alle hatten eine normale Miktion sowie bei fehlender Penisverkrümmung eine normale sexuelle Funktion. Diese Beobachtungen stellen die Notwendigkeit zur rein kosmetischen Korrektur bei anteriorer Hypospadie in Frage.

Goepel et al. stellten in einem Kollektiv von 200 Patienten verschiedenste Formen der Hypospadiekorrektur vor. Bei 27 Patienten mit koronarer Hypospadie wurde die Mathieu-Technik mit einer Erfolgsrate von $56 \%$ verwendet. Aufgrund dieser Ergebnisse wurde diese Technik durch die "island-onlay-flap"-Technik nach Duckett ersetzt, wobei dieses Verfahren geeignet für die penile und distale Hypospadie ist. Die bisherigen Ergebnisse an $22 \mathrm{~Pa}-$ tienten sind gut (1 Fistel, 1 Meatusstenose).

Perovic zeigte die Nachteile der intraoperativen artifiziellen Erektion mit gewaltsamer Dilatation der Schwellkörper, einer nur kurzen Erektionsdauer und der technisch schwierigen Durchführung bei perinealen oder skrotalen Formen der Hypospadie auf. Zur Verringerung dieser Nachteile wurde die physiologische Erektion durch Prostaglandin $\mathrm{E}_{1}(1 \mu \mathrm{g})$ befürwortet und an 607 Patienten durchgeführt. Die Lappenbildung kann in der verlängerten erektilen Phase stattfinden. Der erhöhten intraoperativen Blutungsneigung kann durch Kompression und verkürzter Op-Zeit entgegengewirkt werden. Sehr kritisch wurde der Einsatz bei Kindern vom Auditorium diskutiert. 
Beintker et al. beschäftigten sich mit der Laser-Dopplerflowmetrie an 25 penilen Hautlappen. Gezeigt wurden die Flowunterschiede verschiedener anatomischer Strukturen mit höchsten Raten an Arterien und freiem vaskularisierten Gewebe. Bei kompletter Ischämie fand sich eine Reststase. Ob sich durch den Einsatz dieser Technik die Komplikationsrate von Harnröhrenrekonstruktionen senken läßt wurde offen gelassen, wobei in der Diskussion die routinemäßige Anwendung dieses Verfahrens aufgrund der nur geringen klinischen Aussagekraft in Frage gestellt wurde.

An einem Kollektiv von 85 Patienten wurde von Mersdorf et al. die Notwendigkeit der Harnableitung nach Hypospadiekorrektur diskutiert. Bei distalen Formen der Hypospadie und Korrektur durch proximal gestielten Penisschafthautlappen wurde auf eine Harnableitung verzichtet. Die Fistelrate wurde hierdurch nicht gesenkt. Bei anderen Formen der Hypospadie ist ein "Dripping-Stent" ausreichend. Die Fistelrate liegt im Literaturbereich. In der Diskussion wurde im Auditorium die Notwendigkeit der antibiotischen Prophylaxe z. B. mit Cotrimoxazol hervorgehoben, der Rückschluß zwischen Stent und Fistelbildung allerdings als fraglich dargestellt.

Perovic beschrieb die Bedeutung der Urethralplatte bei der Hypospadiekorrektur. Je nach Schweregrad kann die Urethralplatte intakt belassen, mobilisiert und dabei nicht durchtrennt oder durchtrennt werden. Falls sie nicht durchtrennt wird, ist sie als Dorsalwand bei der "onlay-island"-Technik zu verwenden. Bei sehr stark ausgeprägter Chorda ist eine Durchtrennung unumgänglich. Der Defekt wird dann durch eine Tube-Technik überbrückt. Bei intrinsischer Chorda wurde zusätzlich eine Plikation durchgeführt. Bei 198 Patienten wurden Komplikationen in $5 \%$ der Fälle beobachtet. Die anatomischen, funktionellen und kosmetischen Ergebnisse waren zufriedenstellend. In der Diskussion wurde die Deckung mit reichlich Subkutangewebe zur Senkung der Fistelinzidenz betont.

Westenfelder stellte ein aktuelles Therapiekonzept der Hypospadiekorrektur vor, mit dem die Operation zuverlässig, traumaarm, einzeitig und kosmetisch sowie funktionell befriedigend durchführbar ist. Die einzelnen Schritte umfassen die Diagnostik, die Korrektur und das Zusammenfügen. Wichtig sind die intraoperative Analyse, die individuelle Korrektur, geeignete Techniken, das einzeitige Vorgehen um den 12. Lebensmonat und die stationäre Aufnahme mit der Mutter.
In seinem zweiten Vortrag zeigte Westenfelder das Vorgehen bei schweren Hypospadie-Krüppeln auf. Diese sind durch eine funktionell verschlossene Harnröhre mit meist persistierendem Harnwegsinfekt und begleitenden Komplikationen, exzessive Vernarbungen der lokalen Hautverhältnisse und eine psychische Notfallsituation charakterisiert. Entscheidend sind hier eine Therapiepause von mindestens 6 Monaten, die Rückwandlung zur proximalen Hypospadie, die Infektsanierung, die vorbereitende Korrektur und der anschließende Neuaufbau. Zur Vorbereitung der endgültigen Korrektur kann das Glied mit Andractim-Gel (Dihydrotestosteron-Derivat) vorbehandelt werden. Bei 22 Patienten wurden 2 Strikturen im Meatusbereich sowie 1 Fistel beobachtet. Nach Möglichkeit sollte keine einzeitige Therapie erfolgen.

Abschließend wurde von Kröpfl der Fall eines 28jährigen Patienten mit multiplen Voroperationen bei skrotaler Hypospadie geschildert, bei dem zusätzlich eine sanduhrförmige Einengung der distalen Corpora cavernosa bestand. Die Ausübung des GV war unmöglich. Neben der Hypospadiekorrektur mit Bildung der Neourethra in Onlay-Technik unter Verwendung von Mundschleimhaut wurde das Enge Segment aus den Corpora cavernosa reseziert, die Corpora reanastomosiert und mit einer GoretexProthese armiert sowie an der Glans fixiert. Die Erektion ist postoperativ ungestört, GV möglich.

Dr. med. Stefan Schumacher

Urologische Klinik und Poliklinik der Johannes Gutenberg-Universität Langenbeckstr. 1

55131 Mainz 